University of Massachusetts Amherst

ScholarWorks@UMass Amherst

Chemistry Department Faculty Publication Series

Chemistry

1990

\title{
On-line Pre-concentration of Refractory Elements for Atomiser, Source, Inductively Coupled Plasmas in Atomic Fluorescence Spectrometry (ASIA)
}

Stanley Greenfield

Loughborough University of Technology

Tariq M. Durrani

Loughborough University of Technology

Satilmis Kaya

Loughborough University of Technology

Julian Tyson

University of Massachusetts Amherst

Follow this and additional works at: https://scholarworks.umass.edu/chem_faculty_pubs

Part of the Analytical Chemistry Commons

\section{Recommended Citation}

Greenfield, Stanley; Durrani, Tariq M.; Kaya, Satilmis; and Tyson, Julian, "On-line Pre-concentration of Refractory Elements for Atomiser, Source, Inductively Coupled Plasmas in Atomic Fluorescence Spectrometry (ASIA)" (1990). Analyst. 1400.

Retrieved from https://scholarworks.umass.edu/chem_faculty_pubs/1400 


\title{
On-line Pre-concentration of Refractory Elements for Atomiser, Source, Inductively Coupled Plasmas in Atomic Fluorescence Spectrometry (ASIA)*
}

\author{
Stanley Greenfield, Tariq M. Durrani, Satilmis Kaya and Julian F. Tysont \\ Department of Chemistry, University of Technology, Loughborough, Leicestershire LE11 3TU, UK
}

\begin{abstract}
A simple flow manifold coupled to an atomiser, source, inductively coupled plasmas in atomic fluorescence spectrometry (ASIA) system for the pre-concentration of refractory elements is described. A miniature column of Amberlite IRA-93 anion-exchange resin was used to pre-concentrate refractory elements such as tungsten, molybdenum, zirconium and vanadium as their oxyanions from synthetic aqueous solutions before detection. A study of the parameters that affect the performance of the method is described. The pre-concentrated species were eluted with a stream of ethanolic ammonia directly into the nebuliser of the atomiser plasma. The limits of detection based on a sample volume of $35 \mathrm{ml}$ (sampling time $5 \mathrm{~min}$ at 7 $\mathrm{ml} \mathrm{min}^{-1}$ ) were 65 and $15 \mathrm{ng} \mathrm{ml}^{-1}$ for tungsten and molybdenum, respectively. Relative standard deviations of 4.28 and $5.24 \%$ were obtained for 0.5 and $0.1 \mu \mathrm{g} \mathrm{ml}-1$ concentrations of tungsten and molybdenum, respectively. Further improvements in detection limits were obtained after modification to the instrument; although the main objective of this work was to develop a pre-concentration method applicable to the simultaneous determination of a number of refractory elements, this was not achieved. However, the proposed method could be used for pre-concentration of tungsten and molybdenum.
\end{abstract}

Keywords: Pre-concentration; atomiser source inductively coupled plasma; atomic fluorescence spectrometry; refractory element; tungsten and molybdenum

Atomic fluorescence spectrometry (AFS) has been of considerable interest to analytical atomic spectroscopists for a number of years. ${ }^{1.2}$ The main advantage of this technique over other techniques, particularly inductively coupled plasma atomic emission spectrometry (ICP-AES), is the simplicity of the spectra obtained. This alleviates the problem of spectral interferences to which ICP-AES is prone. Unfortunately, analytical applications of the technique have suffered from the lack of commercial instrumentation. However, a commercial instrument has been introduced by Baird which uses pulsed hollow-cathode lamps as the source of excitation and an ICP as the atomiser. ${ }^{3}$ The instrument used in the work described here $^{4}$ utilises a high-power plasma (source plasma) to excite atomic fluorescence from the atoms formed in a low-power plasma (atomiser plasma).

In general, detection limits for most elements determined using this technique are comparable to, or better than, those in ICP-AES, except for refractory elements. Refractory elements such as tungsten exhibit low sensitivity in the AFS technique, possibly owing to the formation of refractory oxides. The use of carbon-containing species has been recommended 5,6 for the reduction of oxides formed in the atomiser plasma. Carbon can be introduced by passing propane, at low flow-rates, into the injector tube of the atomiser plasma, to produce a long-tailed plasma resembling a flame. Alternatively, organic solvents can be introduced into the atomiser plasma. This leads to an increase in sensitivity but, unfortunately, neither approach provides sufficient sensitivity to permit detection limits comparable to those achievable by ICP-AES.

Pre-concentration of refractory elements prior to their nebulisation is an alternative for improving the sensitivity by this technique. A variety of pre-concentration techniques are available to enhance the sensitivity, including chelating ion-exchange resins, ${ }^{7}$ solvent liquid - liquid extraction, ${ }^{8}$ co-

* Presented at SAC 89, the 8th SAC International Conference on Analytical Chemistry, Cambridge, UK, 30 July-5 August, 1989.

$\uparrow$ Present address: Department of Chemistry, Lederle Graduate Research Tower A, University of Massachusetts, Amherst, MA 01003 , USA.

precipitation $^{9-11}$ and electro-deposition methods. ${ }^{12}$ Recently, Furuta et al. ${ }^{13}$ reported a pre-concentration flow injection system utilising a microcolumn of activated alumina to pre-concentrate molybdenum from sea water prior to determination by ICP-AES.

The aim of this study was to develop a rapid and simple on-line pre-concentration procedure that would allow a number of refractory elements to be determined simultaneously and rapidly.

\section{Experimental}

\section{Apparatus}

The ASIA system consists of two ICPs. The source plasma contained in a Greenfield torch and powered by a Radyne RD150 high-frequency generator $(15 \mathrm{~kW}$ output at $7 \mathrm{MHz}$ ). The atomiser plasma is contained in a long-sleeved Baird torch, powered by a Radyne SC15 high-frequency generator $(2.5 \mathrm{~kW}$ output at $36 \mathrm{MHz})$. A detailed description of the instrument and its operation has been given elsewhere. 5 During the course of this work, modifications to the instrument were made which are listed in Table 1 . The Optica monochromator has been replaced with a Bentham Instruments computer-controlled monochromator with an improved light-gathering power. A single lens is used between the atomiser plasma and the monochromator entrance slits rather

Table 1. Modifications made to the ASIA system

Component

Monochromator

Photomultiplier tube

Stepping motor drive

Power amplifier for SMD

Microcomputer

Peripherals .

Current amplifier

Lock-in amplifier

Display unit
Description

Bentham M300 (computer controlled) EMI $9558 \mathrm{OB}$

Bentham SMD 3B/IEEE

Bentham SMD2

Opus PC II

Hewlett-Packard HP 7470 A and MP-165 printer

Bentham 265HF (programmable)

Bentham 223

Bentham 217 
(a)

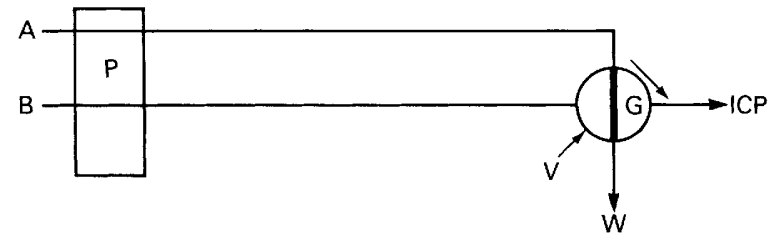

(b)

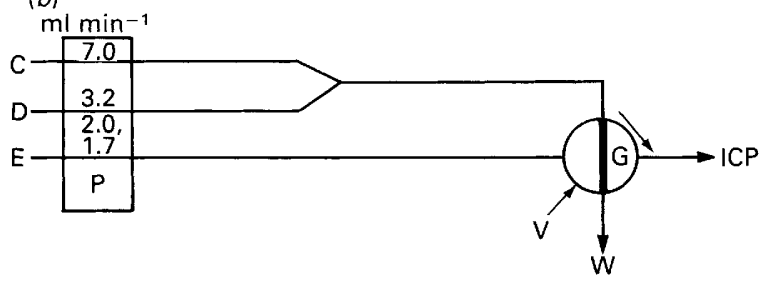

Fig. 1. Pre-concentration manifolds. (a) Single-line manifold: A, sample mixed with buffer at variable flow-rate; $B$, eluent at variable flow-rate; $\mathrm{W}$, waste; $\mathrm{P}$, pump; $\mathrm{V}$, valve; $\mathrm{G}$, column. $(b)$ Dual-line manifold: C, sample; D, buffer; E, eluent

than the original two lenses, which allows greater collection of the fluorescence radiation from the atomiser plasma.

The pre-concentration manifold (Fig. 1) consisted of a Gilson Miniplus 2 (eight-channel) peristaltic pump and an Omnifit six-port injection valve incorporating a laboratorymade glass column $(50 \mathrm{~m} \times 2.3 \mathrm{~mm}$ i.d. $)$ containing anionexchange resin (Amberlite IRA-93, 16-50 mesh). All the connecting tubing consisted of $0.8 \mathrm{~mm}$ i.d. PTFE. Solutions were pumped with Tygon tubing of the appropriate size to obtain the desired flow-rates.

The column was prepared by placing a porous PVC membrane at one end of the column and aspirating a water slurry of the resin into the column until it was filled. Another PVC membrane was placed at the other end of the column and sealed to the plastic connectors using an epoxy resin. The column was placed in the injection loop of the valve so that the sample was loaded in one direction and eluted in the other, which prevented the resin from packing into one end of the column.

\section{Reagents}

All reagents were of analytical-reagent grade. Stock standard solutions of tungsten, molybdenum and vanadium were prepared from sodium tungstate $(\mathrm{BDH})$, molybdenum oxide (BDH) and vanadium oxide (Aldrich), respectively. A stock solution of zirconium ( $\left.995 \mu \mathrm{g} \mathrm{ml}^{-1}\right)$ from Aldrich was used. A series of working standard solutions for each of the four elements were prepared by dilution of the stock standard solutions with de-ionised water.

For the source plasma, a high-concentration solution of tungsten $(40 \%, \mathrm{~m} / \mathrm{v})$ was prepared from dodecatungstosilicic acid (Fisons) dissolved in cold de-ionised water. A $20 \%(\mathrm{~m} / \mathrm{v})$ molybdenum solution was prepared by dissolving molybdenum oxide in the minimum amount of $35 \%$ ammonia solution. A stock solution of vanadium $(1 \mathrm{mg} \mathrm{ml}-1)$ was prepared by dissolving the oxide in $10 \mathrm{ml}$ of concentrated hydrochloric acid. Molybdenum oxide and vanadium oxide were dried at $110^{\circ} \mathrm{C}$ for $2 \mathrm{~h}$ before weighing.

Ethanolic $2.0 \mathrm{~m}$ ammonia solutions of tungsten were prepared by adding a measured volume of $35 \%$ ammonia solution to accurately measured volumes of tungsten stock solution in a calibrated flask, followed by the addition of a calculated volume of de-ionised water and dilution to volume with $99 \%$ ethanol with constant stirring. The ethanolic $2.0 \mathrm{~m}$ ammonia solutions were prepared freshly because solutions containing $80 \%$ or more of ethanol tend to form a precipitate after some time.
The buffer solutions were prepared from ammonium acetate $(\mathrm{BDH})$ and potassium chloride $(\mathrm{BDH})$. The $\mathrm{pH}$ of the ammonium acetate buffer solutions was adjusted to the appropriate value by adding acetic acid or ammonia solution, whereas the $\mathrm{pH}$ of the potassium chloride buffer solutions was adjusted by adding hydrochloric acid or ammonia solution.

\section{Method Development}

A systematic study was carried out to determine the optimum operating conditions of the instrument for the elements studied using an alternative variable search method, ${ }^{14}$ while monitoring the total fluorescence signal. In emission experiments the figure of merit used was the signal to background ratio. The variables included plasma gas flow-rate, observation height and solution flow-rate. The emission lines selected for tungsten, molybdenum, zirconium and vanadium were $400.9,379.8,339.198$ and $310.2 \mathrm{~nm}$, respectively.

The performance of the pre-concentration manifold was studied by investigating the effect of buffer $\mathrm{pH}$, sample and eluent flow-rates and eluent and buffer strengths. Measurements were made on-line for most of the parameters studied in the emission mode using the manifold in Fig. 1(a). The influence of $\mathrm{pH}$ on the retention of the metal species on the column was studied using $0.1 \mathrm{~m}$ potassium chloride $(\mathrm{pH} 1-3)$ and $0.5 \mathrm{M}$ ammonium acetate $(\mathrm{pH} 3.5-8.0)$ buffers. The column efficiency was determined by passing metal solutions through the column at a flow-rate of $1 \mathrm{ml} \mathrm{min}^{-1}$. The column effluents were analysed using the conventional continuous aspiration method. The results obtained were used to calculate the retention efficiency of the column for each of the four elements. The effect of sample flow-rate was studied by passing metal solutions through the column at different flow-rates and analysing the column effluent to determine the percentage retention of the elements. A number of eluents were studied, including nitric acid, hydrochloric acid, sodium carbonate and ammonia at concentrations between 1 and $4 \mathrm{~m}$. Ammonia solutions were optimised for ethanol percentages for measurements made in the fluorescence mode.

\section{Method Performance}

The performance of the method was studied by constructing calibration graphs for molybdenum and tungsten. Standard solution at a flow-rate of $7 \mathrm{ml} \mathrm{min}-1$ was merged with a buffer flowing at $3.2 \mathrm{ml} \mathrm{min}^{-1}$ at the optimum $\mathrm{pH}$. This solution was then passed through the column for $5 \mathrm{~min}$. After the analyte had been deposited on the resin column, elution was effected by switching the valve to allow a stream of $2.0 \mathrm{M}$ ammonia solution in $75 \%$ ethanol at the optimised flow-rate to pass through the column. The valve was then switched to the "load" position and the column was washed with blank solution for $30 \mathrm{~s}$ to allow the column to reach the optimum $\mathrm{pH}$. The procedure was repeated for the next solution.

\section{Results and Discussion}

\section{Method Development}

Fig. 2 shows that for tungsten, quantitative retention was obtained at $\mathrm{pH} 5$ and below, whereas for molybdenum the response was fairly sensitive to $\mathrm{pH}$, as indicated by the sharp maximum at $\mathrm{pH}$ 2.0. For zirconium and vanadium the behaviour of the resin was different, showing quantitative retention at and above $\mathrm{pH} 4.0$ for both elements. This dissimilarity in the behaviour of the resin for the four elements studied can be attributed to the dissimilarity of the metal species formed in the solutions. These results show that $\mathrm{pH} 2.0$ was suitable for the pre-concentration of tungsten and molybdenum. However, $\mathrm{pH} 4.5$ was used for the pre-concentration of tungsten because of the gradual deterioration of the resin at $\mathrm{pH} 2.0$. 


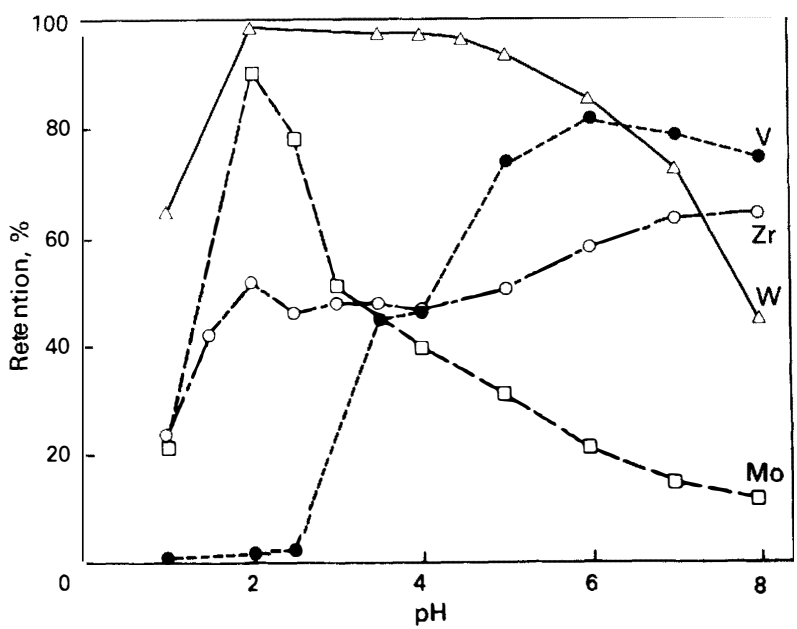

Fig. 2. Effect of varying $\mathrm{pH}$ on metal retention using the manifold in Fig. 1(a)

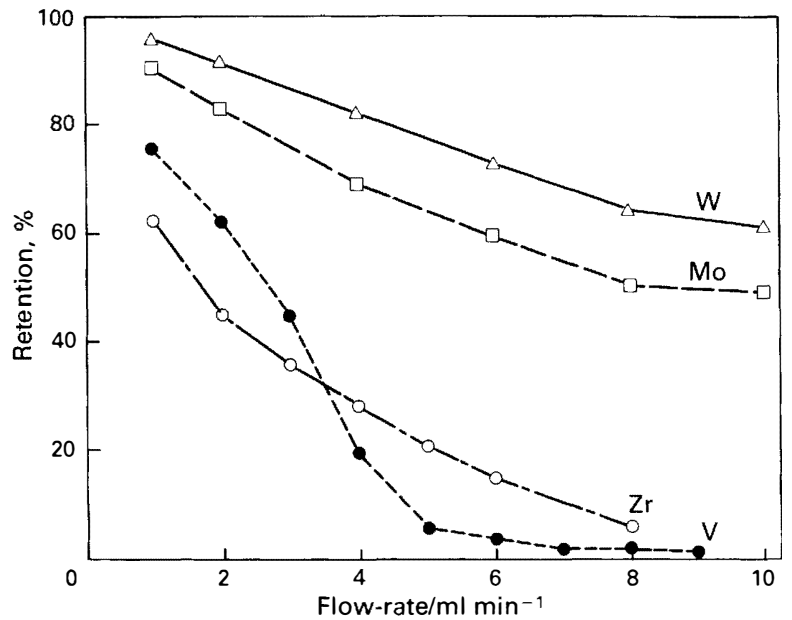

Fig. 3. Effect of varying sample flow-rate on metal retention using the manifold in Fig. 1(a)

The effect of sample flow-rate on the retention of the metal species is shown in Fig. 3. The retention decreases as the flow-rate increases above $1 \mathrm{ml} \mathrm{min}^{-1}$. This behaviour could be due to the kinetic effect relating the retention efficiency with the time the sample is in contact with the resin. Several factors are of value when making projections concerning the dynamic behaviour of resins, including the rate constant and the rate of interaction between the resin and the ions, which is a time-dependent factor. On the basis of the results obtained, these elements could be divided into two groups: (i) tungsten and molybdenum and (ii) zirconium and vanadium. The retention of zirconium and vanadium falls fairly rapidly as the flow-rate of the sample is increased above $1 \mathrm{ml} \mathrm{min}^{-1}$. A possible reason for this behaviour of the resin could be the formation of unstable hydroxo complexes or polymerisation. The clements of the fourth and fifth groups such as zirconium and vanadium tend to hydrolyse, polymerise or precipitate even in fairly acidic solutions; hydrofluoric acid solutions have been recommended ${ }^{15}$ for the formation of fluoro complexes, which are more stable than the hydroxo complexes. However, hydrofluoric acid could not be used with this particular manifold system because the column was made of glass.

The results of the retention efficiency experiments are shown in Fig. 4. The retention of tungsten, molybdenum, zirconium and vanadium falls after loading $3000,700,550$ and $500 \mu \mathrm{g} \mathrm{ml}^{-1}$ of these elements, respectively. The retention

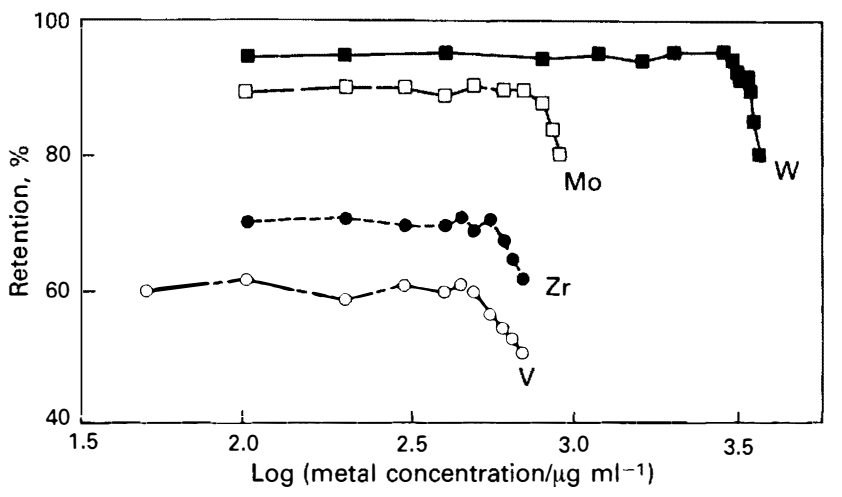

Fig. 4. Retention behaviour of the resin column

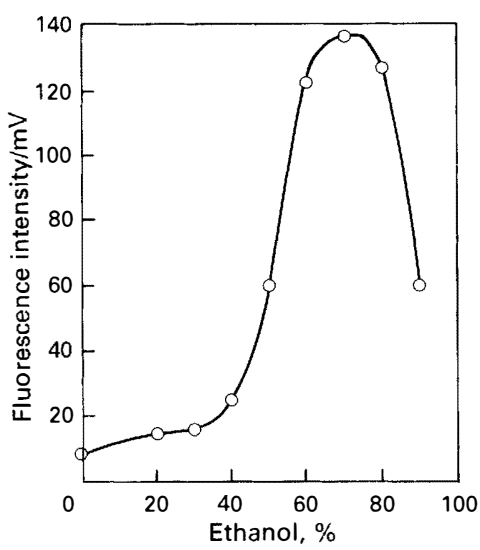

Fig. 5. Effect of percentage of ethanol on tungsten $\left(750 \mu \mathrm{g} \mathrm{ml}^{-1}\right)$ atomic fluorescence signal, while aspirating a $40 \%$ tungsten solution into the source plasma

efficiency calculations were made as recommended by Jacintho et al. 16

Four types of eluents for eluting the retained metal species were investigated. It was found that nitric acid and hydrochloric acid at concentrations up to $4.0 \mathrm{~m}$ were less effective than ammonia solutions in eluting retained metal species from the resin column. The use of sodium carbonate solution resulted in a high blank value, which excluded its further use. A $2.0 \mathrm{M}$ ammonia solution was found to be most suitable in eluting all the retained metal species for measurements made in the emission mode. However, for atomic fluorescence measurements a $75 \%$ ethanolic $2.0 \mathrm{~m}$ ammonia solution was found to be suitable.

As described earlier, special means are employed to maintain refractory elements as free atoms in the plasma tail plume for atomic fluorescence measurements. In this study, ethanol was used to provide the necessary reducing environment. The effect of ethanol on the atomic fluorescence signal of tungsten (Fig. 5) shows that the fluorescence signal increases as the percentage of ethanol increases until the $75 \%$ level is reached, after which a rapid decrease in signal occurs, probably owing to quenching of the fluorescence signal by the carbon species. Subsequently a $75 \%$ ethanolic $2.0 \mathrm{~m}$ ammonia solution was used to elute the sorbed metal ions. When using ethanol in the $2.0 \mathrm{~m}$ ammonia eluent there was no apparent effect on the eluting properties. An advantage of using ethanolic over aqueous ammonia solution was that there was less swelling of the resin column, which avoided any pressure build-up in the flow manifold, ensuring long-term operation of the column without leakage. 
Table 2. Comparison of detection limits obtained by ASIA in the AFS mode ( $\mathrm{g} \mathrm{ml}^{-1}$ )

\begin{tabular}{|c|c|c|c|c|c|c|}
\hline & \multirow[b]{2}{*}{ Element } & \multirow[b]{2}{*}{ Wavclength/nm } & \multicolumn{2}{|c|}{ Previous system } & \multicolumn{2}{|c|}{ Modified system } \\
\hline & & & $\begin{array}{c}\text { Conventional } \\
\text { nebulisation }\end{array}$ & $\begin{array}{c}\text { Pre- } \\
\text { concentration }\end{array}$ & $\begin{array}{l}\text { Conventional } \\
\text { nebulisation }\end{array}$ & $\begin{array}{c}\text { Pre- } \\
\text { concentration }\end{array}$ \\
\hline W & . & 295.6 & 900 & 65 & 428 & - \\
\hline Mo & & 313.3 & 146 & 15 & 63 & 8 \\
\hline
\end{tabular}

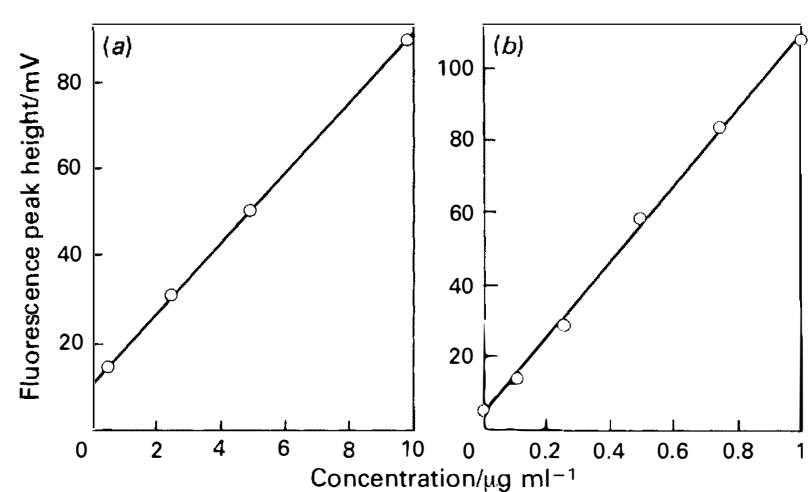

Fig. 6. Calibration graphs for $(a)$ tungsten and $(b)$ molybdenum, while aspirating 40 and $20 \%$ solutions of tungsten and molybdenum, respectively, into the source plasma

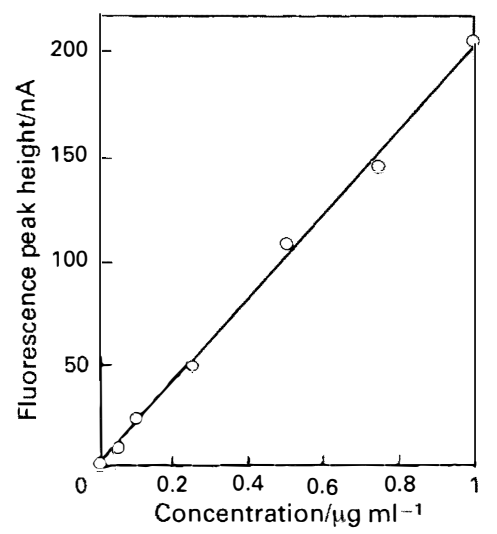

Fig. 7. Calibration graph for molybdenum while aspirating $20 \%$ molybdenum solution into the source plasma

\section{Method Performance}

It was observed in the study of the effect of various parameters on the method performance that only tungsten and molybdenum could be rapidly pre-concentrated. Zirconium and vanadium showed $<5 \%$ retention on the column at a sample flow-rate of $8 \mathrm{ml} \mathrm{min}^{-1}$ and it was therefore considered unnecessary to continue with these elements. Calibration graphs for tungsten and molybdenum are presented in Fig. 6, and show good linearity. The relative standard deviations for tungsten at 10 and $0.5 \mu \mathrm{g} \mathrm{ml}^{-1}$ concentrations were 1.17 and $4.28 \%$, respectively, and those for molybdenum at 1 and 0.1 $\mu \mathrm{g} \mathrm{ml}-1$ were 2.45 and $5.24 \%$, respectively. The limits of detection based on three standard deviations of five replicate measurements of $0.5 \mu \mathrm{g} \mathrm{ml}^{-1}$ of tungsten and $0.1 \mu \mathrm{g} \mathrm{ml}-1$ of molybdenum were 65 and $15 \mathrm{ng} \mathrm{ml}^{-1}$, respectively. After modifications to the instrument, a calibration graph for molybdenum was constructed using the manifold in Fig. 1(b), the results of which are shown in Fig. 7. A detection limit of 8 $\mathrm{ng} \mathrm{ml}-1$ was obtained for a set of five replicate measurements on $50 \mathrm{ng} \mathrm{ml}-1$ molybdenum solution using the procedure described earlier. The precision at this concentration was $4.76 \%$. A comparison of the results obtained using the pre-concentration method and conventional nebulisation is shown in Table 2. Under the recommended conditions, the sampling frequency is 10 samples per hour.

\section{Conclusions}

This study has demonstrated that a pre-concentration manifold incorporating a microcolumn of an anion-exchange resin and subsequent elution of sorbed metal species using an organic solvent medium offers a method for the determination of refractory elements by AFS at the $\mathrm{ng} \mathrm{ml}^{-1}$ level. It may be possible to increase the sensitivity by increasing the sample volume. However, the sampling frequency will be lowered accordingly.

Unfortunately, different conditions for pre-concentration were found to be necessary for different elements and the method would have only limited application to the ASIA work. As a consequence, further work with the proposed method was not pursued. However, the method would be of value if tungsten and/or molybdenum were to be determined by AFS. The manifold could be applicd to other refractory elements that form oxyanions, e.g., boron and chromium, for which the normal sensitivity in AFS is relatively low.

Financial support for T. M. D. from the Ministry of Science and Technology, Government of Pakistan, is gratefully acknowledged.

\section{References}

1. Epstein, M. S., Bayer, S., Bradshaw, J., Voigtman, E., and Winefordner, J. D., Spectrochim. Acta, Part B, 1980, 35, 233.

2. Smith, B. W., Glick, M. R., Spears, K. N., and Winefordner, J. D., Appl. Spectrosc., 1989, 43, 376.

3. Demers, D. R., and Allemand, C. D.. Anal. Chem., 1981, 53, 1915.

4. Greenfield, S., Anal. Proc., 1984, 21, 61.

5. Greenfield, S., and Thomsen, M., Spectrochim. Acta, Part B, $1986,41,677$

6. Greenfield, S., and Thomsen, M., Spectrochim. Acta, Part B. 1985, 40, 1369.

7. Phillips, R. J., and Fritz, J. S., Anal. Chim. Acta, 1980, 121, 225.

8. Kim, C. H., Alexander, P. W., and Smythe, L. E., Talanta, $1975,22,739$.

9. Smith, C. L., Moteoka, J. M., and Willsen, R., Anal. Lett., 1984,17 (A15), 1715.

10. Harda, H., and Kurata, N., Bunseki Kagaku, 1985, 34, 175

11. Akagi, T., Nojiri, Y., Matsui, M., and Haraguchi, H., Appl. Spectrosc., 1985, 39, 662.

12. Henriet, D., De Gelis, D., and Berneron, R., Mem. Etud. Sci. Rev. Metall., 1983, 80 (2), 73.

13. Furuta, N., Brushwyler, K. R., and Hieftje, G. M., Spectrochim. Acta, Part B, 1989, 44, 349.

14. Greenfield, S., Salman, M. S., Thomsen, M., and Tyson, J. F. J. Anal. At. Spectrom., 1989, 4, 55.

15. Nelson, F., Rush, R. M., and Kraus, K. A., J. Am. Chem. Soc., 1960, 82, 339

16. Jacintho, A. O., Zagatto, E. A. G., Bergamin, Fo. H., Krug, F. J., Reis, B. F., Bruns, R. E., and Kowalski, B. R., Anal. Chim. Acta, 1981, 130, 243. 